\title{
Scaling and criticality in a phenomenological renormalization group
}

\author{
Giorgio Nicoletti, ${ }^{1}$ Samir Suweis, ${ }^{1,2}$ and Amos Maritan ${ }^{1}$ \\ ${ }^{1}$ Dipartimento di Fisica “G. Galilei”, Università di Padova, Via Marzolo 8, 35131 Padova, Italy \\ ${ }^{2}$ Padova Neuroscience Center, University of Padova, Padova, Italy
}

(Received 3 January 2020; revised manuscript received 20 March 2020; accepted 14 April 2020; published 8 May 2020)

\begin{abstract}
We present a systematic study to test a recently introduced phenomenological renormalization group, proposed to coarse-grain data of neural activity from their correlation matrix. The approach allows, at least in principle, to establish whether the collective behavior of the network of spiking neurons is described by a non-Gaussian critical fixed point. We test this renormalization procedure in a variety of models focusing in particular on the contact process, which displays an absorbing phase transition at $\lambda=\lambda_{c}$ between a silent and an active state. We find that the results of the coarse graining do not depend on the presence of long-range interactions and, overall, the method proves to be able to distinguish the critical regime from the supercritical one. However, some scaling features persist in the supercritical regime, at least for a finite system, as we see in a contact process above $\lambda_{c}$. Our results provide both a systematic test of the method and insights on the possible subtleties that one needs to consider when applying such phenomenological approaches directly to data to infer signatures of criticality.
\end{abstract}

DOI: 10.1103/PhysRevResearch.2.023144

\section{INTRODUCTION}

The possibility that living systems may be poised at criticality is a fascinating hypothesis [1-3], and in recent years it has been explored in a vast variety of areas [4-7].

Tools from statistical mechanics, such as the renormalization group [8-10], teach us that at criticality the macroscopic, collective behavior of the system is described by a few relevant attributes, such as the embedding dimension of the system and its symmetries, while most the microscopic details of the system become irrelevant. At the critical point the physical properties are determined by a nontrivial fixed point in the space of the possible models. However, in the broad landscape of natural systems one often has to deal directly with data without an explicit model, and the systems are typically finite, so that most of the time it is hard to come up with a definitive answer about whether they are poised near a critical point [3].

Recently, a phenomenological coarse-graining procedure was introduced in $[11,12]$ to deal with the long-range interactions that one reasonably expects in a network of neurons, but of which the full interaction network is not necessarily known. Data from single-neuron recordings, from the hippocampus of a mouse running along a virtual track, were directly analyzed by the authors in order to understand if this coarse-graining procedure (which we recall in Sec. II) drives the system towards a nontrivial fixed point in the renormalization group sense, hence if the neural dynamics is critical and details

Published by the American Physical Society under the terms of the Creative Commons Attribution 4.0 International license. Further distribution of this work must maintain attribution to the author(s) and the published article's title, journal citation, and DOI. independent. Indeed, the brain is probably one of the most impressively complex system we are able to study and the idea that the collective behavior of neurons might emerge from a self-organized critical state has been widely studied in the last year [2,13-15].

One of the first evidences that suggested this hypothesis is the presence of neuronal avalanches that spontaneously occurs in the brain, i.e., during spontaneous activity, that show a spatiotemporal power-law distribution with exponents compatible with those of a mean field branching process [6]. However, this conclusion is highly debated, and the same exponents may stem, for instance, from an underlying noncritical neutral [16] or random [17] dynamics and, in general, the subject is far from being settled.

In this paper we aim to test this phenomenological renormalization group (PRG) method by applying it to a well known nonequilibrium statistical model, the contact process [18]. This model belongs to the universality class of directed percolation and displays an absorbing phase transition, which has been widely studied [19]. Its critical behavior is well understood and the exponents are known from numerical studies, so we shall regard it as a "control case" to investigate the ability of this procedure to extract the relevant information and infer signatures of a critical state in out-of-equilibrium systems.

On a $d$-dimensional hypercubic lattice with nearest neighbor interactions it is sufficient to introduce long-range connections to change the topology, for instance, to that of a small-world network. Hence by simulating the contact process we are able in particular to probe the impact of short- and long-range interactions on the coarse-grained system behavior, and in particular we are able to shed some light on the possible outcomes and interpretations of the emergent fixed point describing the system collective behavior. Along the road we also test the PRG procedure in other models to better characterize its results. 


\section{THE COARSE-GRAINING PROCEDURE}

In this section we briefly describe the coarse-graining procedure introduced in $[11,12]$ that we aim to test $[20]$. The authors propose to build clusters of variables by grouping together neurons that are most correlated, so that the overall correlation structure tends to be preserved.

Let us consider a system (e.g., neural circuit) of $N$ random variables (e.g., neurons activity), whose interaction is unknown. Denoting state variables of the neurons as $\sigma_{i}^{(1)}$ for $i=1, \ldots, N$, where the superscript 1 denotes that we are at the first step of the renormalization procedure, we consider the maximal nondiagonal element of the normalized correlation matrix

$$
c_{i j}=\frac{C_{i j}}{\sqrt{C_{i i} C_{j j}}},
$$

where $C_{i j}$ is the covariance matrix

$$
C_{i j}=\left\langle\sigma_{i}^{(1)} \sigma_{j}^{(1)}\right\rangle-\left\langle\sigma_{i}^{(1)}\right\rangle\left\langle\sigma_{j}^{(1)}\right\rangle,
$$

and $\langle\cdot\rangle$ represents the average over the time series of neural activity. We remove this pair of maximally correlated variables $\left[i, j_{*}(i)\right]$ and repeat the procedure until no other pair remains. The coarse-grained variables are then defined as

$$
\sigma_{i^{\prime}}^{(2)}=\sigma_{i}^{(1)}+\sigma_{j_{*}(i)}^{(1)},
$$

where $i^{\prime}=1, \ldots, N / 2$. We iterate this process, producing clusters of $K=1,2,4, \ldots, 2^{k-1}$ variables. Each one defines a new variable $\sigma_{i}^{(k)}$ as the summed activity of cluster $i$.

Under this coarse-graining procedure in $[11,12]$ the behavior of various quantities is analyzed in order to make some parallels with the behavior of critical systems. In particular, the following observables are studied: the mean variance of the neural activity; the distribution of the individual coarsegrained variables; the spectrum of the covariance matrix; and the mean autocorrelation function.

The mean variance of the activity over the time series is defined as

$$
M_{2}(K)=\frac{1}{N_{k}} \sum_{i=1}^{N_{k}}\left[\left\langle\left(\sigma_{i}^{(k)}\right)^{2}\right\rangle-\left\langle\sigma_{i}^{(k)}\right\rangle^{2}\right],
$$

where $K=2^{k-1}, N_{k}$ is the number of variables after $k$ steps of the coarse-graining procedure, and $N_{k+1}=N_{k} / 2$. If the variables are independent one would obtain a variance scaling as $M_{2}(K) \propto K^{\tilde{\alpha}}$ with $\tilde{\alpha}=1$.

The authors in [12] also suggest to look directly at the probability distributions over a single coarse-grained variable. Since a coarse-grained variable $\sigma_{i}^{(k)}$ is vanishing if and only if all the corresponding $2^{k-1}$ raw variables determining its value are zero, we can split the probability distribution into a contribution of the silent variables and one of the active variables. Hence, we write

$$
\begin{aligned}
P\left(\sigma_{i}^{(k)}\right)= & P_{\text {silence }}(K) \delta\left(\sigma_{i}^{(k)}, 0\right) \\
& +\left[1-P_{\text {silence }}(K)\right] A_{K}\left(\sigma_{i}^{(k)}\right)
\end{aligned}
$$

for some function $A_{K}, K=2^{k-1}$, and where $P_{\text {silence }}$ is the probability that $\sigma_{i}^{(k)}=0$. As suggested by the authors in [12], we can also think of the second term on the right-hand side (r.h.s.) as the probability distribution $P_{\text {activity }}$ of the normalized activity $x_{i}^{(k)}=\sigma_{i}^{(k)} / K$ inside a cluster of $K$ variables, namely

$$
P_{\text {activity }}\left(x_{i}^{(k)}\right):=Z^{-1} A_{K}\left(\sigma_{i}^{(k)}\right),
$$

with $Z$ a proper normalization constant. The effective (reduced) free energy of the system is defined as

$$
F(K)=\log P_{\text {silence }},
$$

which is based on the assumption that the energy of the system is zero when no activity is present. We are interested in its possible scaling $F(K) \sim-K^{\tilde{\beta}}$. For independent variables we expect $\tilde{\beta}=1$.

A scaling behavior of the ranked spectrum of the covariance matrix at the critical point is expected. In fact, when the system is translational invariant then at the critical point the correlation function decays algebraically as $G(\mathbf{x}) \sim$ $|\mathbf{x}|^{-(d-2+\eta)}$, and one can show (see Appendix A) that the eigenvalues of the covariance matrix scale as

$$
\lambda_{r} \sim r^{-(2-\eta) / d},
$$

where $r$ is the rank of $\lambda_{r}$, ordered from the highest to the smallest. If we consider the variables inside the clusters at each coarse-graining step, the highest possible rank $r$ will be given by the number of variables $K$ that make up each cluster. Hence at criticality we should find

$$
\lambda_{r} \propto\left(\frac{K}{r}\right)^{\mu},
$$

with $\mu=(2-\eta) / d$ as a direct consequence of the power-law decay of the correlation function in space.

Finally, the mean autocorrelation function is obtained by

$$
C^{(k)}(t)=\frac{1}{N_{k}} \sum_{i} C_{i}^{(k)}(t)
$$

where

$$
C_{i}^{(k)}(t)=\frac{\left\langle\sigma_{i}^{(k)}\left(t_{0}\right) \sigma_{i}^{(k)}\left(t_{0}+t\right)\right\rangle-\left\langle\sigma_{i}^{(k)}\right\rangle^{2}}{\left\langle\left(\sigma_{i}^{(k)}\right)^{2}\right\rangle-\left\langle\sigma_{i}^{(k)}\right\rangle^{2}} .
$$

Since we are grouping correlated variables, the decay of the autocorrelation is slower in clusters of bigger size. However, in a critical system we might expect dynamical scaling, which would imply a power-law scaling of the autocorrelation times $\tau_{c} \propto K^{\tilde{z}}$.

A different test can be performed by exploiting the fact that, in systems with translational invariance, the Fourier transform of the correlation function $G(\mathbf{k})$ coincides with the eigenvalue spectrum $\lambda_{\mathbf{k}}$ of the covariance matrix. Since coarse graining in momentum space amounts to average over the Fourier modes with small wavelength, we might expect that averaging over low variance contributions in the covariance matrix should lead to an equivalent result. Hence we consider the set of eigenvectors of the covariance matrix $\left\{\mathbf{u}_{r}\right\}$, ordered according to the value of the corresponding eigenvalue, from the the highest to the smallest one, and we introduce the projectors

$$
P_{i j}(K)=\sum_{r=1}^{K} u_{i r} u_{j r},
$$


where $P_{i j}(N)$ is the identity, hence the eigenvectors are orthonormalized. The authors of [11,12] propose to consider a cutoff $\hat{K}<N$, in analogy to the cutoff in momentum space, in such a way that the low variance contributions do not enter the projector (6). Then the coarse-grained variables are defined as

$$
\phi_{i}(\hat{K}):=z_{i}(\hat{K}) \sum_{j} P_{i j}(\hat{K})\left[\sigma_{j}^{(1)}-\left\langle\sigma_{j}^{(1)}\right\rangle\right],
$$

where $z_{i}(\hat{K})$ assures that the coarse-grained variables have unitary variance, i.e., $\left\langle\phi_{i}^{2}(\hat{K})\right\rangle=1$. By means of the YoungEckart theorem [21], the above procedure allows one to find the best decomposition with rank $\hat{K}$ of the original data matrix. In this setting we might once again look directly at probability distributions, namely

$$
P_{\hat{K}}(\phi)=\left\langle\frac{1}{N} \sum_{i=1}^{N} \delta\left[\phi_{i}(\hat{K})-\phi\right]\right\rangle=\frac{1}{N} \sum_{i=1}^{N} \mathbb{P}\left[\phi_{i}(\hat{K})=\phi\right],
$$

as we change the cutoff $\hat{K}$. In fact, a renormalization group transformation typically drives the joint probability towards a fixed point, and if the variables are weakly correlated such fixed point is the one obtained from the central limit theorem [22]. Hence, the authors of [11,12] propose to use this PRG approach to test whether the joint distribution converges towards a non-Gaussian critical fixed point.

\section{THE MODEL}

The contact process $[18,19,23]$ is possibly the simplest nonequilibrium model used to describe the propagation of neural activity on a network $[15,16]$. Hence, it is a proper modeling framework to test the coarse-graining procedure just described.

Consider a collection of $N$ nodes of a given network. Each node can be either active (occupied) or inactive (empty), and we identify its state by means of a binary variable $\sigma_{i}(t)=$ 1,0 respectively. The activity spreads via a nearest neighbors interaction, and it depends on the number of active neighbors $n_{i}(t)=\sum_{j \in\langle i\rangle} \sigma_{j}(t)$, whereas each active site is emptied at a unitary rate. The rates $w\left[\sigma_{i}(t) \rightarrow \sigma_{i}(t+d t) \mid n_{i}(t)\right]$ that define the process for a node with $k_{i}$ neighbors are given by

$$
w\left[0 \rightarrow 1 \mid n_{i}\right]=\frac{\lambda n_{i}}{k_{i}}, \quad w\left[1 \rightarrow 0 \mid n_{i}\right]=1,
$$

where $\lambda$ is the spreading rate.

The configuration with all empty sites is an absorbing state, since the system cannot escape from it. In particular, if $\lambda>\lambda_{c}$ the stationary state is an active fluctuating phase, whereas if $\lambda<\lambda_{c}$ the system eventually gets trapped in the absorbing configuration. Hence, we choose the density $\rho$ of the active sites as an order parameter, while $\lambda$ is the control parameter. In fact, exactly at $\lambda=\lambda_{c}$ the density of active sites undergoes large fluctuations and the system is often close to the absorbing state [24].

The nature of the phase diagram can be readily understood from the mean field approximation

$$
\dot{\rho}=\rho(\lambda-1)-\lambda \rho^{2} .
$$

(a)

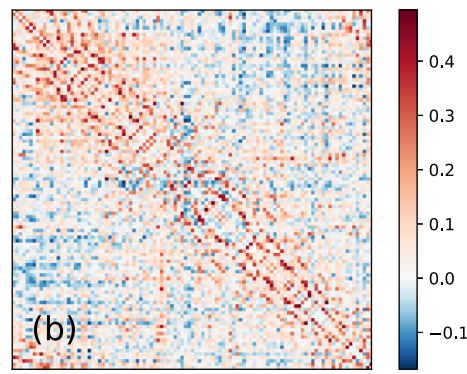

FIG. 1. The correlation matrix for the $2 \mathrm{D}$ contact process at $\lambda=$ $\lambda_{c}$. (a) Correlation matrix of the raw variables. (b) Correlation matrix of clusters of 16 variables. Notice how the coarse graining seems to preserve and unravel the nontrivial correlation structure.

This equation has two stationary solutions: $\rho_{\mathrm{st}}^{v}=0$ and the active state $\rho_{\mathrm{st}}^{a}=(\lambda-1) / \lambda$. The former is stable if $\lambda<1$, and the latter if $\lambda>1$. Hence the mean field critical point is $\lambda_{c}^{\mathrm{MF}}=1$.

The contact process is not exactly solvable even in one dimension, therefore we need to rely on numerical studies. We implement the usual scheme [26]: an occupied site $i$ is randomly chosen, and with probability $1-p_{\lambda}=1 /(1+\lambda)$ the site is emptied. With probability $p_{\lambda}=\lambda /(1+\lambda)$ one of the neighbors is picked at random and, if empty, is occupied. The time is increased by $1 / N_{\text {occ }}$, where $N_{\text {occ }}$ is the number of occupied sites.

We are interested in two different types of interaction network topology to test their effect on the coarse-graining procedure: a two-dimensional (2D) lattice with periodic boundary conditions and a small-world network. The former is a rather standard choice, and the estimated critical point is $\lambda_{c}^{2 \mathrm{D}} \approx$ 1.6488 [19]. On the other hand, the latter setting is more realistic, given the existence of long synaptic connections occurring in a network of neurons.

In general, it would be ideal to have a coarse-graining procedure that works both for short-range and long-range interactions, especially if one needs to deal directly with neural activity data and the specific network architecture is not accessible. In the small-world case the critical point $\lambda_{c}^{\mathrm{SW}}$ depends on the rewiring probability, and it has been studied numerically in [27].

We perform all the simulation with $N=40^{2}$ sites and analyze clusters of size $K=2, \ldots, 256$. In momentum space, we keep up to $N / 128 \approx 12$ eigenvalues, which is less than $1 \%$ of the original modes.

\section{RESULTS}

\section{A. Critical and supercritical cases}

We now consider the contact process in a 2D lattice, both at $\lambda=\lambda_{c}^{2 \mathrm{D}} \approx 1.6488$ and in the supercritical phase at $\lambda=3$. In Fig. 1 we plot the correlation matrix of the critical contact process, before and after the coarse-graining. We will show that the PRG is indeed able to distinguish between these two different phases as the coarse-graining procedure gives indeed results that are rather different. Moreover some subtleties exist that are important to take into account. 

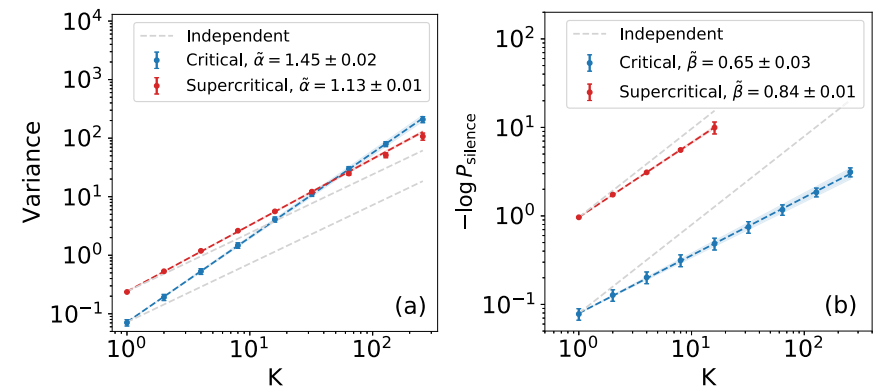

FIG. 2. (a) Scaling of the variance, Eq. (1). (b) Scaling of the free energy, Eq. (3). Both are fitted with the corresponding power laws shown in Sec. II. Notice that both the critical and the supercritical regime show power-law behavior but with different exponents: in particular, the silence probability is smaller and decays much faster in the supercritical case due to the proliferation of the activity. However, we do not find full compatibility between the supercritical contact process and the independent case.

In Fig. 2 we see that in the supercritical regime the exponents of the variance and of the free energy are not exactly compatible to the independent case $\tilde{\alpha}=1=\tilde{\beta}$ [28]. Nevertheless, the profile of the free energy clearly shows that the underlying dynamics is different from the critical state, as for clusters of size $K>32$ the silence probability vanishes in the active phase, a feature characteristic of the supercritical case. Notably, if we compare the critical exponent $\tilde{\beta}=0.65 \pm 0.02$ with the one obtained for real neurons in $[11,12], \tilde{\beta}_{\text {neurons }}=$ $0.893 \pm 0.003$, we see that in the contact process the decay of the silence probability with the cluster size is slightly slower (notice that this does not necessarily mean that real neurons are less active than the sites of a critical contact process, because to quantitatively compare the silence probabilities one should take into account the multiplicative constant in the power-law fit) [29].

Figure 3 shows the correlation structure of the system's quasistationary state. The change in the spectrum of the covariance matrix is more evident, since in the supercritical case the eigenvalues span a smaller set of values. In the
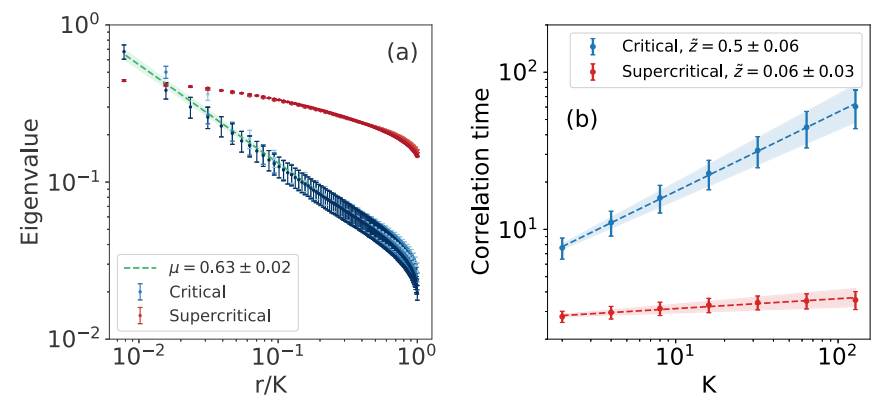

FIG. 3. (a) Scaling of the eigenvalues of the covariance matrix inside clusters of size $K=32,64,128$. (b) Scaling of the autocorrelation times during coarse graining. The distinction among the two phases is rather clear. For the spectrum, in the critical case we find an exponent $\mu=0.63 \pm 0.02$ compatible with the expected value, whereas the eigenvalues show less variability at $\lambda>\lambda_{c}$. The same holds for the autocorrelation times, which follow a power-law behavior at criticality and become negligible above it.
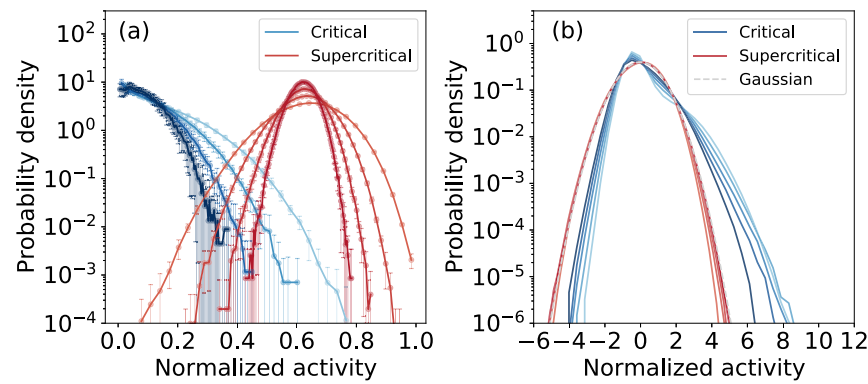

FIG. 4. (a) Evolution of the probability distribution of the nonzero normalized activity during the coarse graining via maximally correlated pairs, as in Eq. (2). (b) Evolution of the probability distribution of the coarse-grained variables in momentum space, as in Eq. (8). We show the results for $K=32, \ldots, 256$ and for $N / 8, \ldots, N / 128$ modes (from brighter to darker color). The distribution in direct space is very different due to the critical contact process being typically close to the absorbing state. In momentum space, the supercritical contact process converges to a Gaussian fixed point in agreement with to the central limit theorem [22], whereas at criticality we see the presence of non-Gaussian tails.

critical case, instead, we find a power-law decay with an exponent $\mu=0.63 \pm 0.02$, with $\mu=(2-\eta) / d$. In real neurons, $[11,12]$ report $\mu_{\text {neurons }}=0.71 \pm 0.06$. We note that, since one of the hyperscaling relations of the contact process yields [30]

$$
\eta=d-2+\frac{\beta}{v_{\perp}}
$$

we expect $\mu \approx 0.6$ in the $2 \mathrm{D}$ contact process from $\beta \approx 0.583$ and $v_{\perp} \approx 0.733$ [19]. This value is compatible with what we find using the PRG procedure. The time-autocorrelation function shows an evident change as well: in the supercritical regime the autocorrelation decays exponentially, whereas at criticality we find a power scaling with an exponent $\tilde{z}=$ $0.50 \pm 0.06$. We note also that in the supercritical regime a power law seems to be present, but the small exponent is compatible with the absence of scaling [31]. Moreover, in Appendix $\mathrm{E}$ we test the robustness of the scaling with respect to the definition of the autocorrelation time and the collapse of the critical time-autocorrelation function.

The evolution of the joint probability distribution of the coarse-grained variables in Fig. 4 shows once more the differences in the underlying dynamics. The most notable result is the convergence in momentum space: for $\lambda>\lambda_{c}^{2 \mathrm{D}}$ the fixed point is Gaussian in accord with the central limit theorem, whereas at $\lambda=\lambda_{c}^{2 \mathrm{D}}$ we see distinct non-Gaussian tails. The last coarse-graining step in momentum space only keeps $N / 128$ modes, so the fact that we still see nontrivial tails is significant.

These results prove to be very stable when we change the underlying topology and we introduce long-range connections by choosing a small-world network. In particular, we implement a Watts-Strogatz model [32] with a rewiring probability $p=0.01$. The critical point is $\lambda_{c}^{\mathrm{SW}} \approx 1.7961$, as given by [27]. We find that all the considerations we made so far hold in the small-world topology as well, and the presence of long-range interactions does not affect the results of the 

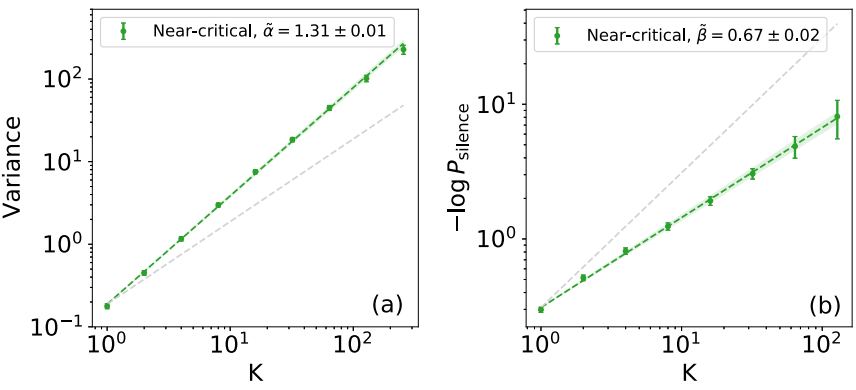

FIG. 5. (a) Scaling of the variance (1) during the coarse graining for $\lambda=\lambda_{\mathrm{nc}}$. (b) Scaling of the free energy (3) at $\lambda=\lambda_{\mathrm{nc}}$. Even if this is a supercritical contact process, the exponents are far from being comparable with the independent case $\tilde{\alpha}=1=\tilde{\beta}$. Overall, the scaling is more similar to the real critical case, see Fig. 2. Notice in particular how the silence probability is nonvanishing even for large clusters.

PRG coarse-graining procedure. In Appendix D we briefly present these results.

As a sanity check, in Appendix D we use a synchronous update algorithm. The results show no difference with respect to the asynchronous one used insofar [33].

\section{B. Persistence of the scaling near a critical point}

A natural question one may ask is how sensible this PRG approach is, i.e., how easy it is to distinguish a truly critical system from a supercritical one. We test this in the contact process by moving the control parameter from the critical point $\lambda_{c}$ to $\lambda_{\text {nc }} \approx 1.1 \lambda_{c}$, that is a $10 \%$ increase.

Notice that, although it is not trivial to define a finite-size critical point [34] for the transition in the contact process [35], at $\lambda_{\text {nc }}$ we do see distinctive features of a supercritical dynamics: in fact, we do not see considerable fluctuations in the density of sites, nor the system constantly approaches the absorbing state as at $\lambda=\lambda_{c}$. Hence, at $\lambda_{\text {nc }}$ the dynamical evolution is significantly supercritical, and we shall refer to this as a near-critical case to distinguish it from the supercritical regime we described before.

In Fig. 5 we see nontrivial scaling behaviors of both the variance and the free energy. If we compare them to Fig. 2, they are arguably more similar to the critical regime rather than the supercritical one [36]. Noticeably, the exponents $\tilde{\alpha}$ and $\tilde{\beta}$ are in between the two cases (i.e., critical and supercritical ones), suggesting that as $\lambda$ smoothly changes from $\lambda=\lambda_{c}$ to $\lambda=+\infty$, the exponents smoothly approach 1 . This result calls for carefulness as the scaling inferred from the PRG of the variance and of the free energy is not related only to the system critical state [37]. Indeed, the difficulty in distinguishing between critical or quasicritical states is confirmed also by other studies (using different approaches) [38].

On the other hand, in Fig. 6 the eigenvalues of the covariance matrix do not display an evident power-law scaling as we change the cluster size, and the scaling of the autocorrelation time function is not significant, especially for larger clusters.

The most convincing results to discriminate between critical and quasicritical state are the joint probability distributions
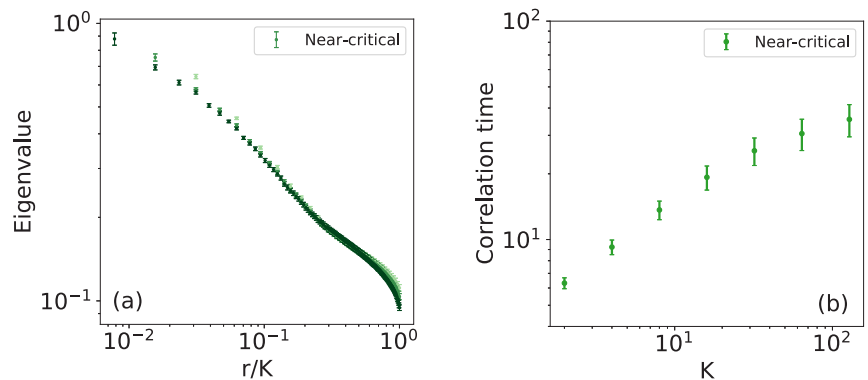

FIG. 6. (a) Scaling of the eigenvalues of the covariance matrix for $K=32,64,128$ (from brighter to darker color). (b) Scaling of the autocorrelation times during the coarse graining for $\lambda \gtrsim \lambda_{c}$. The spectrum of the covariance matrix does not show a power-law decay, and the scaling of the autocorrelation times is not as convincing as the critical case shown in Fig. 3.

[Eqs. (2) and (8)] that we show in Fig. 7, in particular the one in momentum space. We do not see the non-Gaussian tails that we previously found at the critical point, which is expected since away from criticality the variables are much less correlated with one another and they are eventually dominated by the central limit theorem.

Nevertheless, following [17] in Appendix B we introduce a simple model of conditionally independent neurons that shows a nontrivial form of the joint probability distribution along the coarse graining in momentum space although the system is clearly not in a critical state, as in the right panel of Fig. 8. At the same time, for this model the clustering of maximally correlated pairs of variables is able to identify the model as not critical.

In fact one should note that, even though there is no interaction between the neurons themselves, there is an external global parameter that correlates them and hence we do not expect to see a linear scaling of the variance. As a consequence, the fact that in the left panel of Fig. 8 no power-law scaling of the variance is present, should lead one to correctly conclude that this model is not critical.

All the presented results therefore suggest that in order to infer the system state it is crucial not to focus on a single observable, but to analyze all the different coarse-grained quantities.
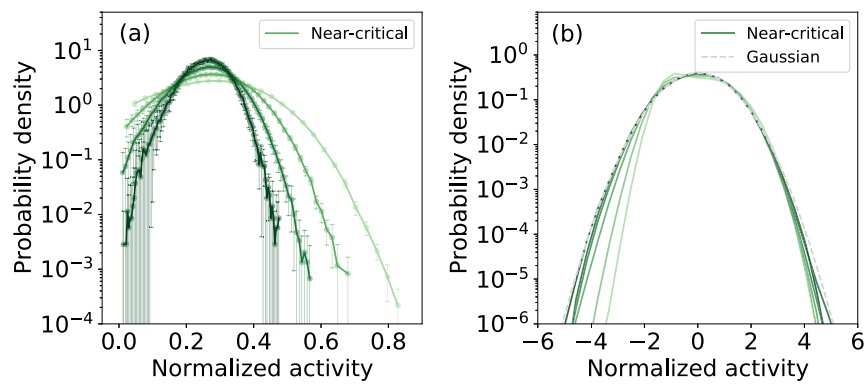

FIG. 7. Evolution of the joint probability during the coarse graining for $\lambda \gtrsim \lambda_{c}$. We show the results (a) for $K=32, \ldots, 256$ and (b) for $N / 8, \ldots, N / 128$ modes. Both of them are comparable with the supercritical case, and in particular in momentum space we do not see the non-Gaussian tails typical of criticality. 


\section{DISCUSSION}

The phenomenological approach introduced in $[11,12]$ has two considerable advantages: it is model independent so it can be applied directly to the data, and it is stable with respect to the presence of long-range interactions. In this work we tested the PRG both in equilibrium models (see Appendix $\mathrm{C}$ for the Ising model), where we expect it to be able to distinguish between critical and noncritical phases, but also in nonequilibrium models, such as the contact process. We have found that the super- and subcritical regimes can be easily recognized, even though the nature of the phase transition is qualitatively different from the one of the Ising model.

At the same time we have highlighted that quasicritical states are difficult to infer, especially if only a subset of physical quantities is analyzed. In nontrivial dynamical models, such as the contact process, the strategy that works best seems to be the one related to the correlation structure. For instance, the presence of non-Gaussian tails in the joint probability distribution of the coarse-grained variables in momentum space, in Figs. 4 and 7, might be a good signature of a possible underlying criticality, but at the same time clustering maximally correlated variables fails as we approach the critical point, Figs. 2 and 5. Interestingly, in considerably simpler models (such as the one of Appendix B) the situation is reversed. Hence, in principle, one needs to study both the approach via maximally correlated pairs and in momentum space. Notably, this is the case of $[11,12]$, hence the claim of the authors that the neuronal dynamics is critical is confirmed by the systematic tests that we have carried out in this work.

We note that the analysis of the binomial model and of the slightly supercritical contact process suggests that this approach might realistically give a set of necessary conditions for criticality, rather than sufficient ones. For instance, the presence of a nontrivial distribution of the coarse-grained variables in momentum space is a necessary condition for criticality because it implies that the underlying variables are strongly correlated, but the convergence to a fixed form in the critical case is hard to assess and calls for particular attention when dealing with experimental data. One might also wonder, inspired by simple noncritical models as the one studied in [17], if there are distinct contributions to the dynamical evolution of the system - an intrinsic one, given by the interaction between the microscopic degrees of freedom, and an extrinsic one, given by some external driver [39]. The binomial model we propose in Appendix B is an archetypal example of the latter, and further research is needed if we want to distinguish the two contributions and, eventually, understand which of them contributes to poising the system at criticality.

Overall we believe that, as it is, this PRG should be considered as a better method to infer the presence of a critical state with respect to typical inference methods based on the identification of avalanches in both size and duration with particular exponents $[3,6,15,40,41]$.

The existence in the data of all the signatures of criticality we have highlighted-such as non-Gaussian tails in the distribution of coarse-grained variables in momentum space-are possibly a powerful and stable indicator to characterize the state of a neuronal network. Extending these methods and test them systematically as in the present work might provide further insights in the understanding of the role of criticality in living systems. For example, recent works suggest that the actual transition in the brain dynamics is not between low and high neural activity states, but rather between an asynchronous and synchronous states [41,42]. An interesting future direction would be to extend the application of this PRG so to characterize different types of critical transitions in terms of the coarse-grained variables.

\section{ACKNOWLEDGMENTS}

S.S. acknowledges UNIPD for BioReACT STARS 2018 grant. A.M. is supported by "Excellence Project 2017" of the Cariparo Foundation.

\section{APPENDIX A: SPECTRAL PROPERTIES OF THE COVARIANCE MATRIX}

Let us show in detail that a power-law spectrum of the covariance matrix is the consequence of the algebraic decay of the spatial correlation function at the critical point, as pointed out in $[11,12]$, at least for a translational invariant system. Consider the covariance matrix

$$
C_{i j}=\left\langle\sigma_{i} \sigma_{j}\right\rangle-\left\langle\sigma_{i}\right\rangle\left\langle\sigma_{j}\right\rangle .
$$

In a system with translational invariance each element of the covariance matrix is given by $C_{i j}=C\left(\mathbf{x}_{i}-\mathbf{x}_{j}\right)$ for some function $C$, whose Fourier transform is given by

$$
\begin{aligned}
C(\mathbf{k}, \mathbf{q}) & =\frac{1}{N} \sum_{i, j} C\left(\mathbf{x}_{i}-\mathbf{x}_{j}\right) e^{-i \mathbf{x}_{i} \cdot \mathbf{k}} e^{-i \mathbf{x}_{j} \cdot \mathbf{q}} \\
& =\delta_{\mathbf{k},-\mathbf{q}} G(\mathbf{k}),
\end{aligned}
$$

where

$$
G(\mathbf{k})=\sum_{n} e^{-i \mathbf{x}_{n} \cdot \mathbf{k}} C\left(\mathbf{x}_{n}\right) .
$$

Hence the covariance matrix has entries given by

$$
C\left(\mathbf{x}_{i}-\mathbf{x}_{j}\right)=\frac{1}{N} \sum_{\mathbf{k}} e^{i \mathbf{k} \cdot\left(\mathbf{x}_{i}-\mathbf{x}_{j}\right)} G(\mathbf{k}),
$$

which means that in Fourier space the covariance matrix is diagonal. In fact, it is easy to show that the eigenvalues are given by the Fourier transform of the correlation function $G(\mathbf{k})$, since

$$
\begin{aligned}
\sum_{\mathbf{x}_{j}} C\left(\mathbf{x}_{i}-\mathbf{x}_{j}\right) e^{i \mathbf{k} \cdot \mathbf{x}_{j}} & =e^{i \mathbf{k} \cdot \mathbf{x}_{i}} \sum_{\mathbf{x}_{j}} C\left(\mathbf{x}_{i}-\mathbf{x}_{j}\right) e^{-i\left(\mathbf{k} \cdot \mathbf{x}_{i}-\mathbf{k} \cdot \mathbf{x}_{j}\right)} \\
& =e^{i \mathbf{k} \cdot \mathbf{x}_{i}} G(\mathbf{k}),
\end{aligned}
$$

hence $e^{i k x}$ is a eigenfunction of eigenvalue $G(\mathbf{k})$.

This has a nontrivial implication for the eigenvalue spectrum of the covariance matrix in a critical system, where we expect the algebraic decay $G(r) \sim r^{-(d-2+\eta)}$. Since the eigenvalues are the Fourier transform of the correlation function, we shall write

$$
\lambda_{\mathbf{k}} \sim \int d^{d} r e^{i \mathbf{k} \cdot \mathbf{r}} r^{-(d-2+\eta)} \sim \frac{1}{|\mathbf{k}|^{2-\eta}} .
$$

If this is a decreasing function of $|\mathbf{k}|$, that is if $\eta<2$, then we consider a ranking of eigenvalues from small momentum 
to large momentum. Hence the highest eigenvalue has rank $r=1$, which implies

$$
\begin{aligned}
r\left[\lambda_{\mathbf{k}}\right] & =\sum_{\mathbf{k}^{\prime}} \mathbb{I}\left[\lambda_{\mathbf{k}^{\prime}}>\lambda_{\mathbf{k}}\right]=\sum_{\mathbf{k}^{\prime}} \mathbb{I}\left[\left|\mathbf{k}^{\prime}\right|<|\mathbf{k}|\right] \\
& \approx L^{d} \int d^{d} k^{\prime} \theta\left(\left|\mathbf{k}^{\prime}\right|<|\mathbf{k}|\right) \\
& \sim(L|\mathbf{k}|)^{d} .
\end{aligned}
$$

This implies that the eigenvalues of the covariance matrix decay as a power law of their rank, namely

$$
\lambda_{r} \sim \frac{1}{r^{\mu}},
$$

where $\lambda_{1} \geqslant \lambda_{2} \geqslant \cdots \lambda_{N}$ and $\mu=(2-\eta) / d$.

\section{APPENDIX B: MODEL OF CONDITIONALLY INDEPENDENT NEURONS}

The convergence of the joint probability distribution in Eq. (8) is related to the spectrum of the covariance matrix, but one should be careful when considering its relation with criticality. Consider $N$ random variables $\left(\sigma_{1}^{t}, \ldots, \sigma_{N}^{t}\right)$. At each time $t$ the distribution of the $i$ th variable, which we can think of as a neuron that can be either active or inactive, is a simple binomial distribution with parameter $\xi_{i}(t)$. However, we consider the case in which also $\boldsymbol{\xi}(t)=\left(\xi_{1}(t), \ldots, \xi_{N}(t)\right)$ is itself a random variable distributed according to some distribution $p(\xi)$, so that the $N$ neurons are conditionally independent. We can think of this case as the one of neurons that follow a common external dynamics, represented by $p(\xi)$, but otherwise show no intrinsic dynamical features.

The probability that a neuron is either active or inactive is then a binomial distribution conditioned to the value of $\xi_{i}(t)$, that is

$$
p\left[\sigma_{i}^{t} \mid \xi=\boldsymbol{\xi}(t)\right]= \begin{cases}\xi_{i}(t), & \sigma_{i}^{t}=1, \\ 1-\xi_{i}(t), & \sigma_{i}^{t}=0,\end{cases}
$$

and the each neuron is described by the joint probability $p\left(\sigma_{i}^{t}, \boldsymbol{\xi}\right)=p\left[\sigma_{i}^{t} \mid \boldsymbol{\xi}=\boldsymbol{\xi}(t)\right] p(\boldsymbol{\xi})$.

Since the coarse-graining procedure depends on the equaltime covariance of the neurons $\operatorname{cov}\left(\sigma_{i}, \sigma_{j}\right)=C_{i j}$, we need the marginal probabilities

$$
p\left(\sigma_{i}^{t}\right)= \begin{cases}\int d \xi_{i}^{*} \xi_{i}^{*} p\left(\xi_{i}^{*}\right)=\left\langle\xi_{i}\right\rangle, & \sigma_{i}^{t}=1, \\ \int d \xi_{i}^{*}\left(1-\xi_{i}^{*}\right) p\left(\xi_{i}^{*}\right)=1-\left\langle\xi_{i}\right\rangle, & \sigma_{i}^{t}=0,\end{cases}
$$

and

$$
p\left(\sigma_{i}^{t}, \sigma_{j}^{t}\right)= \begin{cases}\left\langle\xi_{i} \xi_{j}\right\rangle, & \sigma_{i}^{t}=1, \sigma_{j}^{t}=1, \\ \left\langle\xi_{i}\right\rangle-\left\langle\xi_{i} \xi_{j}\right\rangle, & \sigma_{i}^{t}=1, \sigma_{j}^{t}=0, \\ \left\langle\xi_{j}\right\rangle-\left\langle\xi_{i} \xi_{j}\right\rangle, & \sigma_{i}^{t}=0, \sigma_{j}^{t}=1, \\ 1+\left\langle\xi_{i} \xi_{j}\right\rangle-\left\langle\xi_{i}\right\rangle-\left\langle\xi_{j}\right\rangle, & \sigma_{i}^{t}=0, \sigma_{j}^{t}=0 .\end{cases}
$$

If we now use the latter marginal probability we immediately find that, if $i \neq j$, the first contribution to the covariance matrix of the neurons is given by

$$
\begin{aligned}
\left\langle\sigma_{i} \sigma_{j}\right\rangle_{p\left(\sigma_{i}, \sigma_{j}\right)} & =\sum_{\sigma_{i}, \sigma_{j}=0,1} \sigma_{i} \sigma_{j} p\left(\sigma_{i}, \sigma_{j}\right) \\
& =p\left(\sigma_{i}=1, \sigma_{j}=1\right)=\left\langle\xi_{i} \xi_{j}\right\rangle .
\end{aligned}
$$
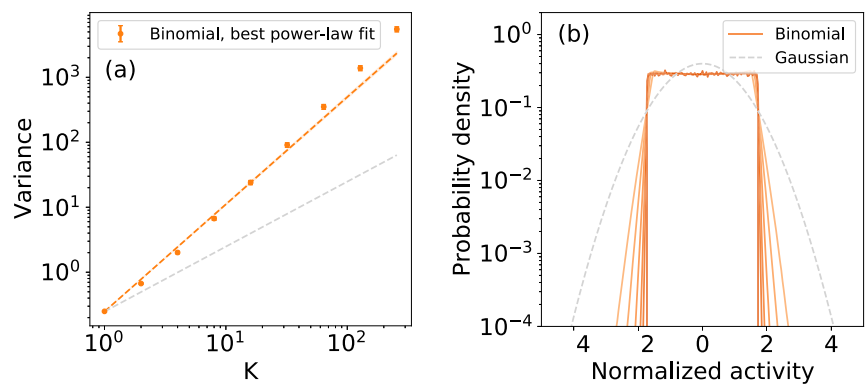

FIG. 8. (a) Failure of the scaling of the variance in the binomial model. (b) The non-Gaussian distribution resulting from the procedure in momentum space.

If instead $i=j$, we shall use the former marginal probability

$$
\left\langle\sigma_{i} \sigma_{i}\right\rangle_{p\left(\sigma_{i}\right)}=\sum_{\sigma_{i}=0,1} \sigma_{i} p\left(\sigma_{i}\right)=\left\langle\xi_{i}\right\rangle .
$$

Hence, even if the neurons are not correlated, their covariance is not vanishing but depends on the covariance of $p(\xi)$,

$$
C_{i j}= \begin{cases}\left\langle\xi_{i} \xi_{j}\right\rangle-\left\langle\xi_{i}\right\rangle\left\langle\xi_{j}\right\rangle, & i \neq j, \\ \left\langle\xi_{i}\right\rangle\left(1-\left\langle\xi_{i}\right\rangle\right), & i=j .\end{cases}
$$

Let us consider the simple case of $\xi_{i}=\xi_{j} \forall i, j$, so that at each time all the neurons fire with the same probability $\xi$, and take $p\left(\xi_{i}\right)$ to be a uniform distribution. In this case the covariance matrix is simply

$$
C_{i j}=a \delta_{i j}+b\left(1-\delta_{i j}\right),
$$

with $a=1 / 4$ and $b=1 / 12$. The eigenvalues of this matrix are given by

$$
\begin{aligned}
& \lambda_{1}=a+(N-1) b, \quad m=1, \\
& \lambda_{2}=a-b, \quad m=N-1,
\end{aligned}
$$

where $m$ is the corresponding multiplicity. Therefore, there are $N-1$ eigenvalues with the same value.

The eigenvector associated to the highest eigenvalue is $1 / \sqrt{N}(1, \ldots, 1)^{T}$, but there is no obvious choice for the other eigenvectors in Eq. (6) because the ranking is illdefined. However, from a numerical standpoint the spectrum of the covariance matrix will not be degenerate, so if we simulate the model we can try to apply the procedure regardless.

As we can see from Fig. 8 the joint probability does not converge to a Gaussian, even though there is nothing critical about the underlying dynamics. Hence the proposed coarse graining in momentum space fails for a simple set of conditionally independent binomial variables, albeit it seemed to be the most promising procedure for the supercritical contact process in the vicinity of the critical point. On the other hand, and perhaps not surprisingly, in this model the proposed coarse-graining procedure via maximally correlated variables does work: in fact, since the off-diagonal elements of the covariance matrix are all equal we are randomly pairing neurons together and no scaling property emerges.

This simple model shows once more how careful one should be when employing these kind of procedures. All 

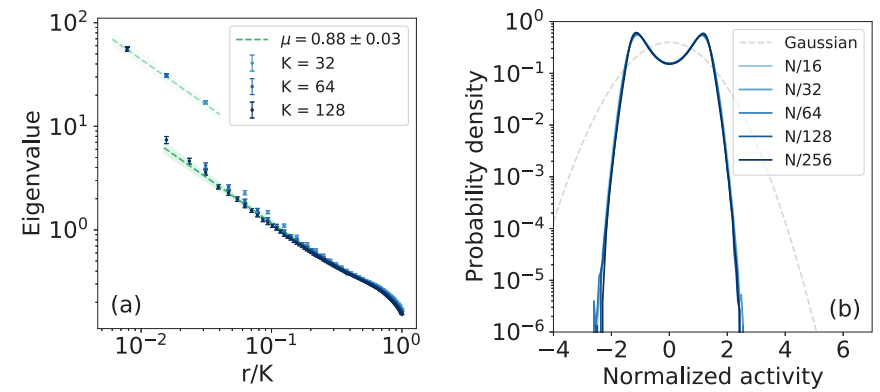

FIG. 9. (a) Scaling of the eigenvalue at the critical temperature of the Ising model. Notice that a dominant eigenvalue is present, because the model starts to order, but it scales in the same way as the rest of the spectrum. (b) Convergence to a non-Gaussian fixed form of the distribution of coarse-grained variables in momentum space, Eq. (8).

in all, the two approaches combined seem to work well, in the sense that a system might really be critical if both of them indicate the presence of an underlying scale invariance. However, when we take them individually they might point in the wrong direction.

\section{APPENDIX C: ISING MODEL}

We also test the coarse-graining procedure in the 2D Ising model, where the PRG builds clusters of maximally correlated spins, by simulating the model at different temperatures using the Wolff algorithm [43]. We do not show all the results explicitly for the sake of brevity, but they are in line with what one might expect.

In the disordered phase at $T>T_{c}$ the coarse graining drives the system towards a behavior that is comparable with the one of independent random variables, very much like one would expect from a usual block-spin transformation in real space.

For $T<T_{c}$, instead, the behavior of coarse-grained variables resembles the one of a perfectly ordered system, where all the spins tend to be aligned.

However, as we lower the temperature we see a nontrivial effect due to the spontaneous symmetry breaking that occurs at the transition. In fact, the Ising model in its ordered phase is essentially low dimensional, as shown in [44], in the sense that one single eigenvalue eventually dominates the spectrum of the covariance matrix at low temperature.

In order to take this into account, we consider separately the behavior of the highest eigenvalues and the rest of the spectrum. We find that at criticality they both scale with the same exponent $\mu=0.88 \pm 0.03$ and only the multiplicative constant differs, see the left panel of Fig. 9. The value is perfectly compatible with the value $\mu=7 / 8$ one gets from the exact solution. Moreover, in the right panel we see that the joint distribution (8) clearly converges to a non-Gaussian form.

Hence, in this case of an equilibrium phase transition with spontaneous symmetry breaking, this procedure does identify two distinct phases [45].
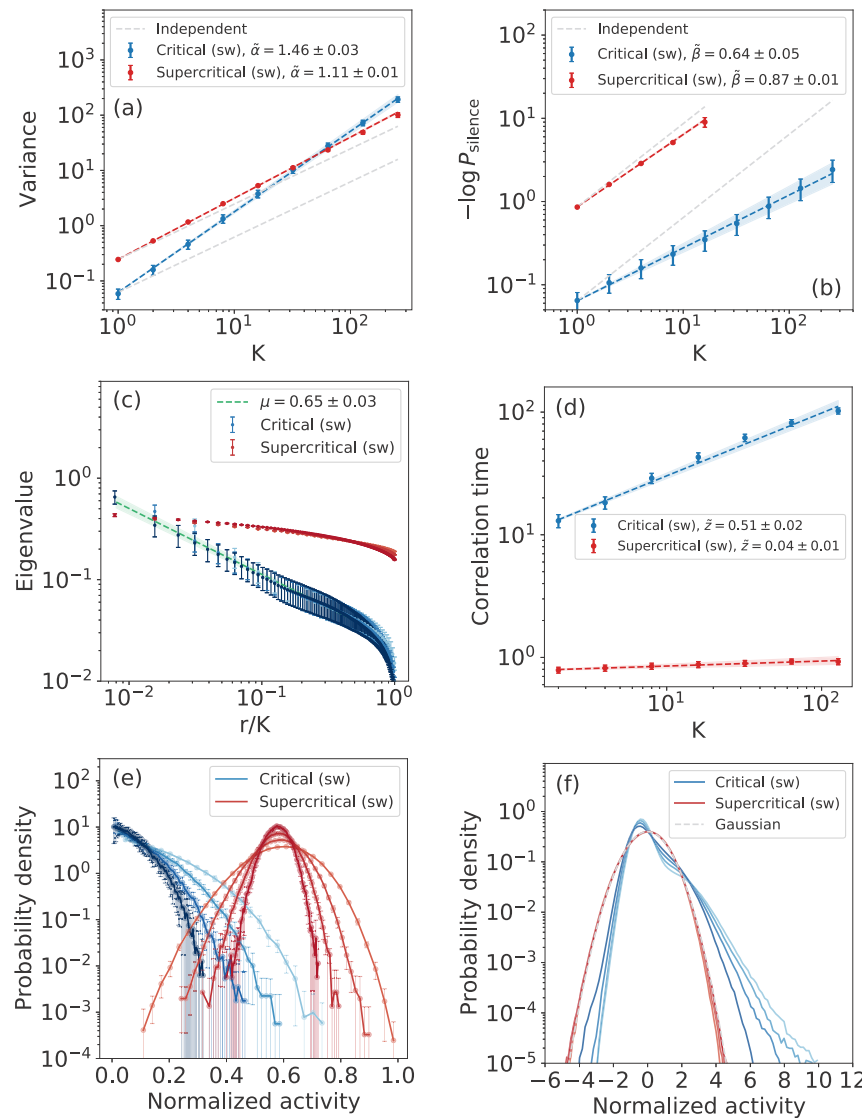

FIG. 10. Results for the contact process in a small network, simulated with a synchronous update algorithm. (a) Scaling of the variance; (b) scaling of the free energy; (c) scaling of the eigenvalues; (d) scaling of the autocorrelation times; (e) joint probability distribution of normalized activity; and (f) joint probability distribution in momentum space.

\section{APPENDIX D: CONTACT PROCESS IN A SMALL-WORLD NETWORK}

As stated in the main text, the results we find do not depend on the choice of the simulation algorithm and, most importantly, the method works even in the presence of longrange interaction. Hence, in this Appendix we briefly present the result for the contact process in a small-world simulated with a synchronous update algorithm. In Fig. 10 we see that all the consideration made in the main text for the $2 \mathrm{D}$ contact process with asynchronous updates hold in this case as well.

These results underline the fact that the method proposed by $[11,12]$ is not sensible to the presence of long-range interaction, since it does not depend on the underlying spatial structure to begin with.

\section{APPENDIX E: AUTOCORRELATION TIMES}

Estimating the autocorrelation time might be challenging due to sampling effects. In order to check the robustness of the scaling we find in Fig. 3, we perform a longer simulation of the 2D critical contact process and we implement three different strategies to evaluate the autocorrelation time. 


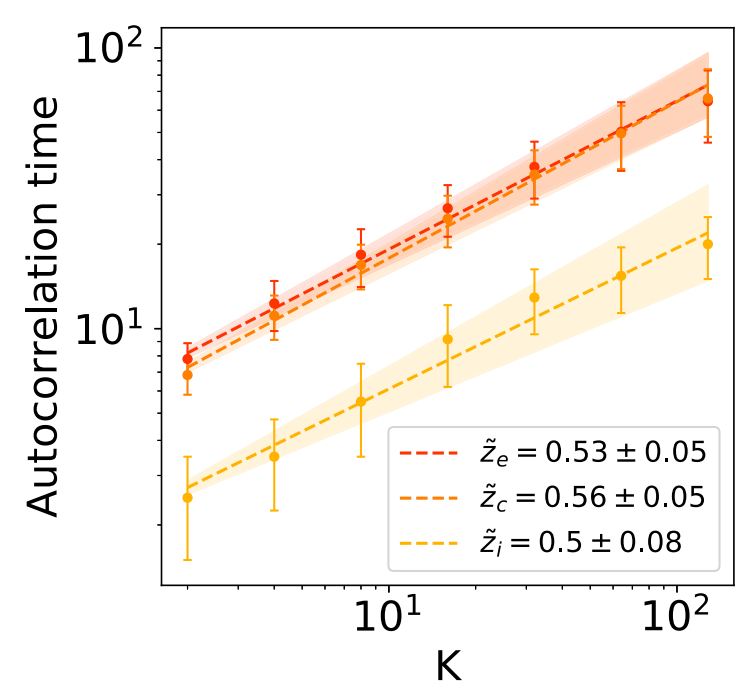

FIG. 11. The scaling of the autocorrelation times in the critical contact process is robust with respect to the definition of the autocorrelation time itself.

In particular, we estimate the exponential correlation time $\tau_{e}$, the integrated correlation time $\tau_{i}$ as defined in [46], and the definition $\tau_{c}$ proposed by Cavagna et al. in [47]. In Fig. 11 we see that the scaling exponent is compatible for all the three estimates, and even though the integrated correlation time is particularly noisy it only differs by an irrelevant multiplicative
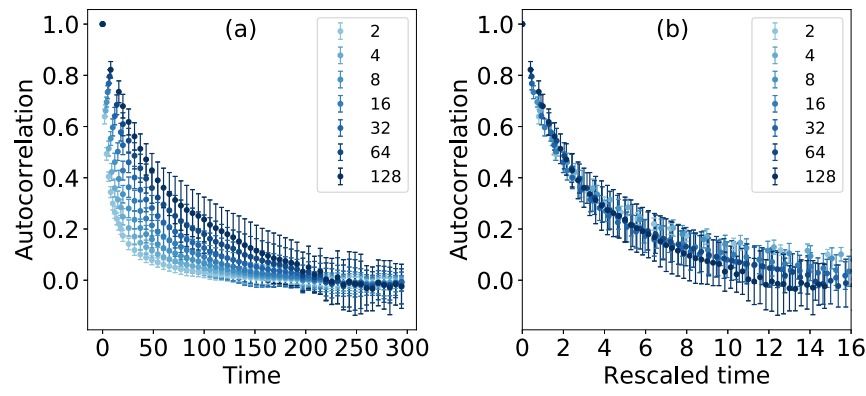

FIG. 12. If we rescale the times by $t \rightarrow t / \tau$, with $\tau$ being the autocorrelation time, the time-autocorrelation functions at different $K$ (a) collapse into a single curve (b).

constant. Hence the scaling we find proves to be robust with respect to the definition of the autocorrelation time.

Moreover, as proposed in [11,12], if we rescale the time by $t \rightarrow t / \tau$ the autocorrelation functions at different $K$ collapse in the same curve at criticality as in Fig. 12. This is typically associated with dynamical scaling, which is expected at the critical point of the contact process.

Notice that, on the contrary, in the supercritical regime the autocorrelation's decay is exponential, and hence we do not see a meaningful change of the the autocorrelation function as we change $K$. In fact, in Fig. 3 in the main text we see a scaling of the autocorrelation times that is compatible with a constant.
[1] T. Mora and W. Bialek, Are biological systems poised at criticality? J. Stat. Phys. 144, 268 (2011).

[2] J. Hidalgo, J. Grilli, S. Suweis, M. A. Muñoz, J. R. Banavar, and A. Maritan, Information-based fitness and the emergence of criticality in living systems, Proc. Natl. Acad. Sci. 111, 28 (2014).

[3] M. A. Muñoz, Colloquium: Criticality and dynamical scaling in living systems, Rev. Mod. Phys. 90, 031001 (2018).

[4] O. Kinouchi and M. Copelli, Optimal dynamical range of excitable networks at criticality, Nat. Phys. 2, 348 (2006).

[5] T. Mora, A. M. Walczak, W. Bialek, and C. G. Callan, Maximum entropy models for antibody diversity, Proc. Natl. Acad. Sci. 107, 12 (2010).

[6] J. M. Beggs and D. Plenz, Neuronal avalanches in neocortical circuits, J. Neurosci. 23, 35 (2003).

[7] A. Cavagna, A. Cimarelli, I. Giardina, G. Parisi, R. Santagati, F. Stefanini, and M. Viale, Scale-free correlations in starling flocks, Proc. Natl. Acad. Sci. 107, 26 (2010).

[8] S.-k. Ma, Modern Theory of Critical Phenomena, Advanced Book Classic (Perseus Books, Cambridge, Massachusetts, 2000).

[9] J. J. Binney, N. J. Dowrick, A. J. Fisher, and M. Newman, The Theory of Critical Phenomena: An Introduction to the Renormalization Group (Oxford University Press, Oxford, 1992).

[10] N. Goldenfeld, Lectures on Phase Transitions and the Renormalization Group (Addison-Wesley, Reading, MA, 1992).
[11] L. Meshulam, J. L. Gauthier, C. D. Brody, D. W. Tank, and W. Bialek, Coarse Graining, Fixed Points, and Scaling in a Large Population of Neurons, Phys. Rev. Lett. 123, 178103 (2019).

[12] L. Meshulam, J. L. Gauthier, C. D. Brody, D. W. Tank, and W. Bialek, Coarse-graining and hints of scaling in a population of 1000+ neurons, arXiv:1812.11904.

[13] D. R. Chialvo, Emergent complex neural dynamics, Nat. Phys. 6, 744 (2010)

[14] G. Tkačik, T. Mora, O. Marre, D. Amodei, S. E. Palmer, M. J. Berry II, and W. Bialek, Thermodynamics and signatures of criticality in a network of neurons, Proc. Natl. Acad. Sci. 112, 11508 (2014).

[15] R. P. Rocha, L. Koçillari, S. Suweis, M. Corbetta, and A. Maritan, Homeostatic plasticity and emergence of functional networks in a whole-brain model at criticality, Sci. Rep. 8, 15682 (2018).

[16] M. Martinello, J. Hidalgo, A. Maritan, S. di Santo, D. Plenz, and M. A. Muñoz, Neutral Theory and Scale-Free Neural Dynamics, Phys. Rev. X 7, 041071 (2017).

[17] J. Touboul and A. Destexhe, Power-law statistics and universal scaling in the absence of criticality, Phys. Rev. E 95, 012413 (2017).

[18] T. E. Harris, Contact interactions on a lattice, Ann. Probab. 2, 6 (1974).

[19] J. Marro and R. Dickman, Nonequilibrium Phase Transitions in Lattice Models (Cambridge University Press, Cambridge, 1999). 
[20] We will use as much as possible the same notations introduced in $[11,12]$.

[21] C. Eckart and G. Young, The approximation of one matrix by another of lower rank, Psychometrika 1, 211 (1936).

[22] G. Jona-Lasinio, Renormalization group and probability theory, Phys. Rep. 352, 4 (2001).

[23] M. Henkel, H. Hinrichsen, and S. Lübeck, Non-Equilibrium Phase Transitions. Volume 1: Absorbing Phase Transitions (Springer, Berlin, 2009).

[24] Indeed one can prove that the critical contact process dies out with probability 1 [25]. A nonvanishing survival probability is achieved only in the supercritical regime $\lambda>\lambda_{c}$.

[25] C. Bezuidenhout and G. Grimmett, The critical contact process dies out, Ann. Probab. 18, 4 (1990).

[26] R. Dickman, Reweighting in nonequilibrium simulations, Phys. Rev. E 60, R2441(R) (1999).

[27] R. Ferreira and S. Ferreira, Critical behavior of the contact process on small-world networks, Eur. Phys. J. B 86, 462 (2013).

[28] One might wonder whether the scaling does persist for all the values of $K$. For instance, we might try to fit only the variance for $K \geqslant 16$. In the critical case we find an exponent $\tilde{\alpha}_{16}^{(\mathrm{c})}=1.43 \pm 0.05$, which is compatible with $\tilde{\alpha}^{(\mathrm{c})}$ and hence suggests that the scaling is consistent at different values of $K$. In the supercritical regime we find $\tilde{\alpha}_{16}^{(\mathrm{sc})}=1.08 \pm 0.03$ which, albeit lower, is still compatible with $\tilde{\alpha}^{(\mathrm{sc})}$ within the error. However, this might suggest a breakdown of the scaling in the supercritical case that we do not see at this scale because it occurs at larger values of $K$. Since such values are typically inaccessible in experiments and simulations, we conclude that the scaling of variance is not particularly informative. The fact that we do not see full compatibility with the independent case might be due to the fact that in the supercritical regime the fluctuations are not fully uncorrelated, and possibly this method tends to overestimate them.

[29] In principle, one might wonder why the apparent exponent of a supercritical contact process is similar to the exponent found in real neuronal data. However, we think that this might well be a numerical coincidence as the fundamental point is that in the supercritical regime of the contact process the scaling fails for large $K$, since the silence probability vanishes in the active phase, a fact that is not observed in real data of neural activity.

[30] R. Dickman and M. M. de Oliveira, Quasi-stationary simulation of the contact process, Physica A 357, 134 (2005).

[31] A constant autocorrelation time across different cluster sizes fits, with the same significance, the data, hence no relevant scaling feature seems to be present in the supercritical regime.

[32] D. Watts and S. H. Strogatz, Collective dynamics of small world networks, Nature (London) 393, 440 (1998).

[33] In the case of the asynchronous update, where at most one site is changed at each step, one should carefully consider that the algorithm induces a spurious correlation between subsequent configurations. Hence we do not keep all the configurations to perform averages but we subsample them so to select only uncorrelated configurations. Once this is taken into account, the results of synchronous and asynchronous updates are equivalent.

[34] A. E. Ferdinand and M. E. Fisher, Bounded and inhomogeneous Ising models. I. Specific-heat anomaly of a finite lattice, Phys. Rev. 185, 832 (1969).

[35] Indeed, the finite size contact process eventually reaches the absorbing configuration, at all $\lambda[19]$.

[36] As in the previous case, we might try to fit only points at larger values of $K$, for instance $K \geqslant 16$. We find that the exponents does get smaller, $\tilde{\alpha}_{16}^{(\mathrm{NC})}=1.24 \pm 0.06$, but once again remains compatible with $\tilde{\alpha}^{(\mathrm{NC})}$. As before, this suggest that we see a nontrivial scaling that might only disappear at very large values of $K$. Given that these are the typical values one can deal with, the scaling of the variance cannot be ruled out for this near-critical system.

[37] The fact that both the variance (1) and the free energy defined in (3) show a power-law behavior both at $\lambda_{c}$ and at $\lambda>\lambda_{c}$ might be a sign that criticality is not a necessary condition for these quantities to scale as a power law. In particular, in the case of the free energy one should note that usually it is the singular part of the free energy that shows scaling, whereas with this PRG we cannot distinguish it from the nonsingular part.

[38] V. Priesemann, M. Wibral, M. Valderrama, R. Pröpper, M. Le Van Quyen, T. Geisel, J. Triesch, D. Nikolić, and M. H. Munk, Spike avalanches in vivo suggest a driven, slightly subcritical brain state, Front. Syst. Neurosci. 8, 108 (2014).

[39] P. S. Swain, M. B. Elowitz, and E. D. Siggia, Intrinsic and extrinsic contributions to stochasticity in gene expression, Proc. Natl. Acad. Sci. 99, 12795 (2002).

[40] J. Hesse and T. Gross, Self-organized criticality as a fundamental property of neural systems, Front. Syst. Neurosci. 8, 166 (2014).

[41] A. J. Fontenele, N. A. P. de Vasconcelos, T. Feliciano, L. A. A. Aguiar, C. Soares-Cunha, B. Coimbra, L. Dalla Porta, S. Ribeiro, A. J. Rodrigues, N. Sousa, P. V. Carelli, and M. Copelli, Criticality between Cortical States, Phys. Rev. Lett. 122, 208101 (2019).

[42] S. di Santo, P. Villegas, R. Burioni, and M. A. Muñoz, LandauGinzburg theory of cortex dynamics: Scale-free avalanches emerge at the edge of synchronization, Proc. Natl. Acad. Sci. 115, E1356 (2018).

[43] U. Wolff, Collective Monte Carlo Updating for Spin Systems, Phys. Rev. Lett. 62, 361 (1989).

[44] L. Wang, Discovering phase transitions with unsupervised learning, Phys. Rev. B 94, 195105 (2016).

[45] One should note that since the Ising variables are $\sigma_{i}= \pm 1$, we cannot define in the same way a silence probability and the corresponding free energy (3).

[46] J. Goodman and J. Weare, Ensemble samplers with affine invariance, Commun. Appl. Math. Comput. Sci. 5, 65 (2010).

[47] A. Cavagna, D. Conti, C. Creato, L. Del Castello, I. Giardina, T. Grigera, S. Melillo, L. Parisi, and M. Viale, Dynamic scaling in natural swarms, Nat. Phys. 13, 914 (2017). 